Report No. LPT Orsay 00-25,UNIL-IPT/00-05

\title{
Baryogenesis from 'electrogenesis' in a scalar field dominated epoch
}

\author{
Michael Joyce \\ LPT, Université Paris-XI, Bâtiment 211, F-91405 Orsay Cedex, France \\ E-mail: Michael.Joyce@th.u-psud.fr \\ Tomislav Prokopec \\ Université de Lausanne, Institut de Physique Théorique, BSP, CH-1015 Lausanne, Suisse \\ E-mail: Tomislav.Prokopec@ipt.unil.ch
}

\begin{abstract}
Scalar fields can play a dominant role in the dynamics of the Universe until shortly before nucleosynthesis. Examples are provided by domination by a kinetic mode of a scalar field, which may be both the inflaton and the late time 'quintessence', and also by more conventional models of reheating. The resultant modification to the pre-nucleosynthesis expansion rate can allow solely an asymmetry in right handed electrons to produce a net baryon asymmetry when reprocessed by the anomalous $B+L$ violating processes of the standard model. The production of such a source asymmetry - what we term 'electrogenesis' - requires no additional $B$ or $L$ violation beyond that in the standard model. We consider a specific model for its generation, by a simple perturbative out-of-equilibrium decay of Higgs like scalar fields with CP-violating Yukawa couplings to the standard model leptons. We show that, because of the much enhanced expansion rate, such a mechanism can easily produce an adequate asymmetry from scalars with masses as low as $1 \mathrm{TeV}$. Kinetic mode domination is strongly favoured because it evades large entropy release which dilutes the asymmetry. We also discuss briefly the effect of the abelian hypercharge anomaly.
\end{abstract}




\section{Introduction}

Until a few years ago cosmology with scalar fields was almost synonymous with cosmological inflation. Recently there has been an enormous upsurge in interest in the possibility that scalar fields can play an important role in the dynamics of the Universe at recent epochs, mainly due to the observations of the apparent magnitudes of distant supernovae [1] which may be explained by the presence of such a component [2]. In this context it is certainly interesting to consider what the role of such fields can be at other epochs, and in particular how their behaviour between the end of inflation and their reappearance today might influence cosmology in the intervening period. This question is also related to the 'fine-tuning' problem associated with such scenarios: how is it that such a field can give a significant contribution to the energy density today starting from a natural set of initial conditions after inflation? This apparent problem is in fact resolved in a wide class of potentials [3, 4] which generically have the property that in some part of the potential they may support modes which are dominated by the kinetic energy of the scalar field, so that their energy density scales away faster than that in the radiation, i.e. $\rho_{\phi} \propto a^{-n}$, with $4<n \leq 6$, where $a$ is the scale factor. In principle there is no reason why such modes cannot initially dominate over the radiation component, and in certain specific models this is realized. The main important observational constraint is that such domination must terminate by the nucleosynthesis epoch, when the expansion law must be that given by radiation domination with the standard model degrees of freedom. There may be an additional contribution which, conservatively, must be less than about $20 \%$ of the total [5].

More generally we can consider the question of the cosmology of the Universe between the end of an inflationary epoch and the entry into radiation domination before nucleosynthesis. For a transition from a scalar field dominated cosmology to occur the energy in the scalar field must either decay (directly or indirectly) into standard model particles - as in standard reheating scenarios [6] - or it must red-shift away more rapidly than the radiation. Or some combination of the two can occur. In the former case any scaling less rapid than that during inflation $\left(n>2\right.$, or equivalently an equation of state $p_{\phi}=w_{\phi} \rho_{\phi}$, with $\left.w_{\phi}>-1 / 3\right)$ can be envisaged, with the case $n=3$ corresponding to the most standard reheating during 
the oscillation of the inflaton about the minimum of a quadratic potential. There is a continual release of entropy until the radiation dominated epoch, leading to a dilution of most relevant physical quantities sourced during the scalar dominated phase. In the latter case, which corresponds to domination by the kinetic energy of a homogeneous scalar field (or equivalently to an equation of state $p_{\phi}=w_{\phi} \rho_{\phi}$, with $w_{\phi}>1 / 3$ ) the scalar field simply redshifts away until it becomes the sub-dominant component. There is no entropy release, and correspondingly a coherent energy remains in the scalar field which, given an appropriate potential (the 'self-tuning' potentials of [3], or the 'tracking' potentials of [4]) can become relevant again at late times [7, 8, 9].

In [10, 8] we have considered in a generic way the effect of a change in the expansion rate prior to nucleosynthesis on models of electroweak baryogenesis on the sphaleron bound and the 'no-go' theorem for electroweak baryogenesis in the case of a second order phase transition. As concrete realizations of such cosmologies we considered models which go through an epoch after inflation - which, following [10] we termed 'kination' - of domination by a kinetic mode of a scalar field. This occurs most naturally in a model in which the universe 'reheats' not by the decay of the inflaton, but by gravitational particle creation at the end of the inflationary epoch [13, 7]. In a recent paper [14] it has been observed that, for low (sub-electroweak) reheat temperatures in more traditional models of reheating - in which the inflaton decays while oscillating in a mode with matter scaling after inflation - the effects discussed in [10, 8] on electroweak cosmology also result. There is in this case an even larger relative boost to the expansion rate (see below), but a very large entropy release which tends to undo any of the enhancing effects of the greater expansion rate. In 15 one of us (TP) has considered the general case of a decaying inflaton evolving in a mode scaling as $1 / a^{n}$, and shown that, while the same larger boost to the expansion rate occurs as in the $n=3$ case of [14], the entropy release problem is greatly reduced as the kinetic mode $n=6$ limit is attained.

Here we concentrate on another aspect of such alternative cosmologies, which is a simple consequence of the observation which has been made in [16, 8, 14]: Because of the enhanced

\footnotetext{
${ }^{1}$ The effects on dark matter freeze-out can be inferred from the work of [11, 12], who studied mainly modifications associated with anisotropy in the expansion.
} 
expansion rate, the right-handed electrons of the standard model may remain out of equilibrium until a temperature below the electroweak phase transition. It is well known that asymmetry in right-handed electrons - because of their late equilibration time - may be important from at least two points of view:

- Since right-handed electrons couple to other particles in the standard model with only an extremely small Yukawa coupling, they remain out of equilibrium in an expanding Universe until relatively late - in the standard radiation dominated cosmology until $T \gtrsim 20 \mathrm{TeV}$ [17, 18]. A pre-existing baryon asymmetry can survive the effect of standard model anomalous processes - which violate $B+L$ and are unsuppressed until the electroweak phase transition - only if there are non-zero CP-odd conserved global charges when they are operative. In the absence of such charges the equilibrium attained will be $\mathrm{CP}$ invariant with zero baryon number. As noted in [18] above 20 20TeV right electron number $e_{R}$ is in fact such an effective charge, and as a result other global charges like $B-L$ can be violated until close to this scale. This leads [18] to a very significant reduction in the bounds on $B-L$ violating interactions in grand unified theories with the structure appropriate for them to generate baryon asymmetry. Here the consequences are much simpler and more dramatic: If the $e_{R}$ remain out of equilibrium all the way until the electroweak scale, a baryon number will result from this due to the $B+L$ violating processes. When the electroweak scale is reached this baryon number will simply be frozen when the $B+L$ violating processes abruptly switch off. This will be the case irrespective of whether there is primordial $B$ or $L$ (or $B-L$ ), and irrespective of whether these charges are violated or conserved. Just like in the case of electroweak baryogenesis all the non-trivial physics required is in principle present in the standard model. The problem of baryogenesis then becomes posed as what we will refer to as 'electrogenesis', the generation of the source right handed electrons prior to the time at which the $B+L$ violating processes become suppressed. It is this process which we discuss below.

- The effective conservation of $e_{R}$ in the early Universe due to the fact that its perturbative decay channel is out of equilibrium is not exact, because the $e_{R}$ charge has an axial

\footnotetext{
${ }^{2}$ The scale quoted in [18] is $10 \mathrm{GeV}$. The increase by a factor 2 is due to a tighter bound on the Higgs mass.
} 
anomaly under the U(1) of hypercharge. There are no degenerate vacua as in the non-abelian case, but there are finite energy modes of the U(1) field with Chern-Simons number which can 'eat' the charge. In fact, as discussed in [16, 19] this leads to an instability at finite density to the formation of long wavelength modes of hypermagnetic field. When these modes come inside the horizon they can evolve during the time in which the right electron number is without its perturbative decay channel. Here this scenario will be modified as a result of the change in the expansion rate, since the perturbative channel does not come into play until the electroweak scale, at which time a first order phase transition may produce the turbulence needed to amplify the produced seed magnetic fields.

\section{Scalar fields and the expansion rate after inflation}

The inflationary solutions for scalar fields represent only one part of a much wider range of possible behaviours of the energy density in the zero modes of scalar fields. The full range can be characterized by the equation of state for a (real) scalar field which is determined by the relative weight of the kinetic and potential energy (see [10, 8] for a discussion):

$$
p_{\phi}=w_{\phi} \rho_{\phi}, \quad w_{\phi}=\frac{\frac{1}{2} \dot{\phi}^{2}-V(\phi)}{\frac{1}{2} \dot{\phi}^{2}+V(\phi)}, \quad \rho_{\phi} \propto a^{-3\left(w_{\phi}+1\right)} .
$$

The limit of potential energy domination gives inflation, with $\rho_{\phi} \approx$ const, while the opposite limit of complete kinetic energy domination gives the most rapid possible red-shifting of the energy to be $\rho_{\phi} \propto 1 / a^{6}$. While inflation is associated with flat potentials (satisfying "slowroll' conditions), the latter limit is associated with steep potentials]. A particularly useful 'yard-stick' of flatness/steepness is the simple exponential potential

$$
V_{\exp }(\phi)=M_{P}^{4} e^{-\lambda \phi / M_{P}}
$$

where $M_{P}=1 / \sqrt{8 \pi G} \approx 2.4 \times 10^{18} \mathrm{GeV}$ is the reduced Planck mass (and the origin of $\phi$ has been chosen to give the simple normalization). This potential in fact has an attractor solution 20] for any $\lambda^{2}<6$ in which the energy density scales as $1 / a^{\lambda^{2}}$, and as $1 / a^{6}$ for $\lambda^{2}>6$. A

\footnotetext{
${ }^{3}$ The exception is a flat direction with no associated potential energy e.g. a Goldstone direction associated with a broken exact global symmetry, which only has pure kinetic modes.
} 
potential with a varying slope, e.g. the inverse power-law potential of [21, 22] $V \sim M_{P}^{4+\alpha} / \phi^{\alpha}$ then supports a kinetic mode at small $\phi$, but an inflationary type (or 'quintessence') mode at large values of the field. Alternatively an oscillating mode about the minimum of a potential $V \sim \lambda_{\alpha} \phi^{\alpha}$ gives a broad range of scalings with $\rho_{\phi} \propto a^{-6 \alpha /(\alpha+2)}$ [23, producing thus the familiar matter scaling when $\alpha=2$ and radiation scaling when $\alpha=4$.

In [8] we discussed several ways in which a period of kinetic mode domination (which, following [10, we termed 'kination') could come about after inflation't. We considered only the case in which the relevant field (the 'kinaton') did not decay itself, and discussed two possible sources for the radiation in the Universe: The entropy associated with particle creation during the de Sitter phase (see below), or a more conventional source in the decay of a distinct inflaton field. In the latter case specific conditions need to be satisfied by the 'kinaton' field to allow it to dominate over the energy produced by the inflaton, whereas in the former the kinaton and the inflaton are one field and the domination by the kinetic mode for a period is a built-in and necessary feature.

Our concern in this paper is not the inflationary model building aspect of the problem, but rather the problem of 'electrogenesis' in this kind of cosmology, as well as in the more conventional reheating models discussed in [14, 15]. For the sake of clarity and simplicity we limit ourselves here to two definite and simple models with scalar field dominance continuing until temperatures just above the nucleosynthesis scale, exemplifying these two types of different cases:

- Model (A): The inflaton rolls after inflation into a steep potential in which the field rolls in a kinetic mode, so that the energy density scales as $1 / a^{n}$ where $n>4$ (see Figure 1). The field is assumed to be very weakly coupled and the only radiation present is the very sub-dominant component due to particle creation at the end of the preceeding de Sitter phase. The latter has a characteristic energy density $H_{I}^{4}$, where $H_{I}$ is the expansion rate at the end of inflation, so that initially $\rho_{\text {rad }} / \rho_{\phi} \sim\left(H_{I} / M_{P}\right)^{2}$. Provided the inflaton scales faster than radiation it will become subdominant at a subsequent time and the transition to radiation domination is achieved without any decay of the field [13, 7]. Requiring that

\footnotetext{
${ }^{4}$ Such kinetic modes have also recently been used to propose a solution to the cosmological moduli problem [24.
} 


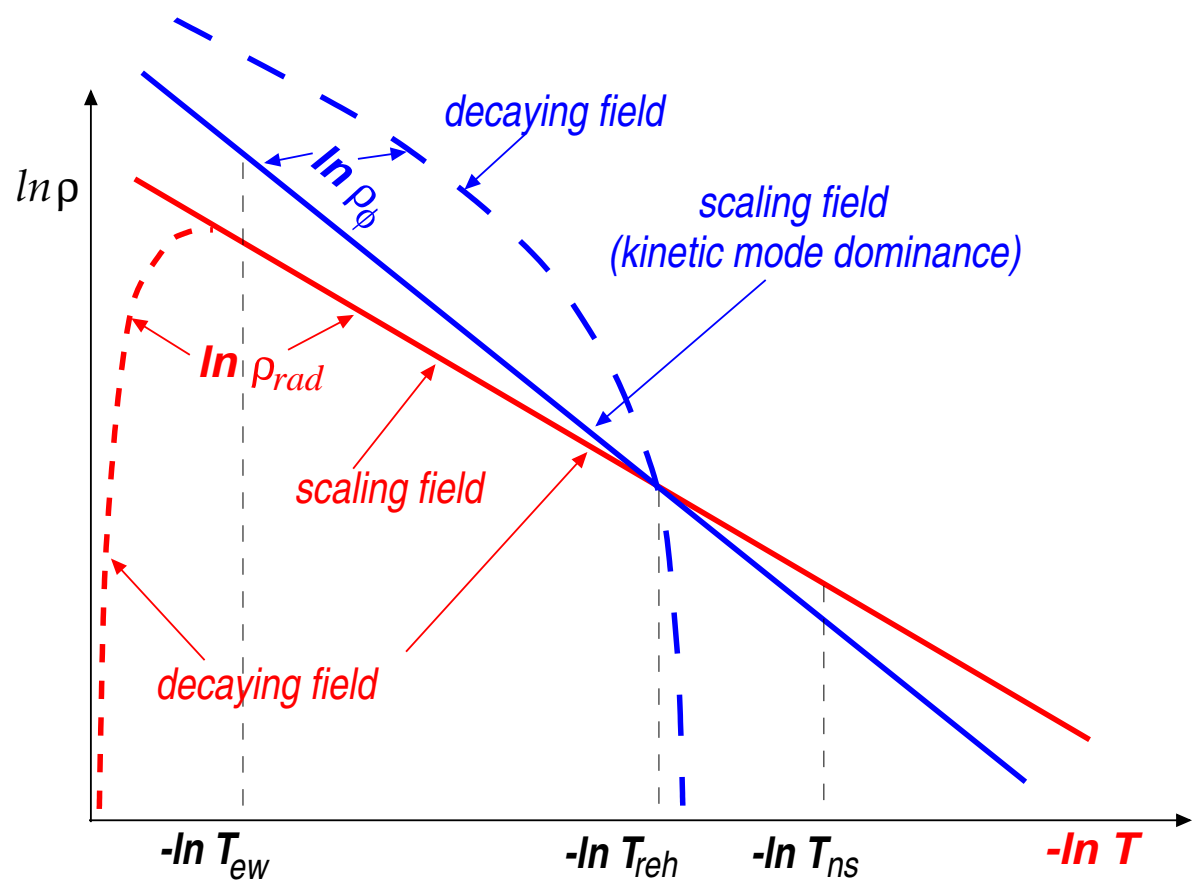

Figure 1: Evolution of energy density in radiation and the dominant scalar field as a function of temperature. Two cases are illustrated: $\operatorname{Model}(A)$ in which the dominant scalar component scales faster then radiation, but does not decay (solid lines), and Model (B) in which the scalar field decays (dashed lines).

this transition occurs before nucleosynthesis gives an absolute lower bound on $H_{I}$, which for a pure (or almost) kinetic mode scaling as $1 / a^{6}$ results is $H_{I} \gtrsim 10^{7} \mathrm{GeV}$ (see [8]). As noted by Spokoiny [7], for an appropriate potential the field can again dominate in a slowly scaling mode at late times. This kind of model has been dubbed 'quintessential inflation' and studied in more detail in [9, 25] (see also [26]).

Taking the reheat temperature $T_{\text {reh }}$ to be defined f as that when $\rho_{\phi} \simeq \rho_{\text {rad }}$, it is easy to

\footnotetext{
${ }^{5}$ Note that in these models the Universe is strictly speaking not 'reheated' at all - the entropy is left behind at the end of the de Sitter phase and the important process is the red-shifting away of the dominant energy in the inflaton. Here we adopt the standard definition of 'reheat temperature' as used in standard reheating models. In [8] we used 'reheat temperature' to mean the temperature of the radiation when it first thermalizes, which is far higher $\left(\sim 0.1 H_{I}\right)$ than the 'reheat temperature' as defined here. What we now call $T_{\text {reh }}$ is denoted $T_{k, \text { end }}$ ('end of kination') in [8].
} 
infer 0 that above this temperature we have

$$
H=H_{\mathrm{rad}}\left(\frac{T}{T_{\mathrm{reh}}}\right)^{\frac{n-4}{2}},
$$

where $H_{\mathrm{rad}} \simeq 1.4 \times 10^{-16}\left(T^{2} / 100 \mathrm{GeV}\right)$ is the standard radiation dominated evolution of the expansion rate, and $n$ gives the scaling of the energy density in the dominant scalar mode $\rho_{\phi} \propto 1 / a^{n}$, with clearly the largest enhancement of the expansion rate for the limit $n=6$. The constraint that the energy density in the scalar field be less than about $20 \%$ at nucleosynthesis requires that $T_{\text {reh }} \gtrsim 5^{1 /(n-4)} T_{\mathrm{ns}}$ (and $T_{\mathrm{ns}}=1 \mathrm{MeV}$ ). Here we are interested in the case when right electrons are out of equilibrium at the electroweak scale, which corresponds therefore to the upper bound

$$
T_{\text {reh }}<T_{\text {ew }}\left(\frac{H_{\mathrm{ew}}}{\Gamma_{e_{R}}}\right)^{\frac{2}{n-4}}
$$

which for the optimum case $(n=6)$ becomes

$$
T_{\text {reh }}<T_{\text {ew }} \frac{H_{\text {ew }}}{\Gamma_{e_{R}}} \sim \frac{T_{\text {ew }}}{200} \sim 0.5 \mathrm{GeV},
$$

where we have made use of the fact that the interaction rate for right electrons through their Yukawa coupling is $\Gamma_{e_{R}} \approx 10^{-13} x_{e_{R}}^{2} T$ [18, and we took $x_{e_{R}} \equiv m_{H} / 2 T_{\text {ew }} \sim 0.5$ correspondinding to the current lower bound on the Higgs mass $m_{H}$. In terms of the expansion rate the bound (5) corresponds to the requirement of a boost by about 200 times in the expansion rate at the electroweak scale relative to that in the standard radiation dominated cosmology.

- Model (B): The inflaton evolves after inflation into a potential, in which it rolls or oscillates, scaling as $1 / a^{n}$ with $6 \geq n \geq 3$. The dominant source of entropy comes from the decay of the inflaton, which is however sufficiently weakly coupled that reheating occurs between the electroweak scale and the nucleosynthesis scale. The energy density-temperature dependence for this case is illustrated in Figure 11. The phase we are discussing corresponds to the 'preheating' phase [27] of inflationary models with the usual mechanism of reheating from inflaton decay, either in an oscillatory mode (with $n=3$ for a $\phi^{2}$ potential) or a

\footnotetext{
${ }^{6}$ For simplicity we neglect here and elsewhere the small reheating factors associated with particle decouplings.
} 
rolling mode. We assume here for simplicity perturbative reheating, but note that the nonperturbative decay channels of narrow resonance may also be considered. A realization of the latter with a rolling mode is given by the 'NO' models of [28].

It is quite easy to show [15] that in Case $(B)$ the expansion rate as a function of the temperature is independent of the equation of state (11), i.e. the following universality in scaling in the expansion rate holds

$$
H=\frac{5-3 w_{\phi}}{6} \frac{\rho_{r}}{\Gamma_{\phi} M_{P}}=H_{\mathrm{rad}}\left(\frac{T}{T_{\mathrm{reh}}}\right)^{2},
$$

which implies that, for a reheat temperature below the electroweak transition, the expansion rate is enhanced by $\left(T / T_{\text {reh }}\right)^{2}$ with respect to the standard rate $H_{\text {ew }} \equiv H_{\text {rad }}\left(T_{\text {ew }}\right)$. The condition that the right electrons remain out of equilibrium until the electroweak scale is in this case

$$
T_{\text {reh }}<T_{\text {ew }}\left(\frac{H_{\text {ew }}}{\Gamma_{e_{R}}}\right)^{\frac{1}{2}} \sim \frac{T_{\mathrm{ew}}}{15} \sim 5 \mathrm{GeV}
$$

which again corresponds to the same minimal boost in the expansion rate by a factor of about 200. The extra increase in the expansion rate as a function of temperature compared to the first case is due to the 'leaking' of the scalar field energy into the radiation. Note that the lower bound on $T_{\text {reh }}$ is in this case $T_{\text {reh }} \gtrsim 2 \mathrm{MeV}$.

Our interest here finally is in the ratio of baryon number to entropy, and so will need to include the dilution effect of this entropy production subsequent to the scale $T_{\text {dec }}$ at which the baryon number, or in fact the source for it, right electron number is produced. As

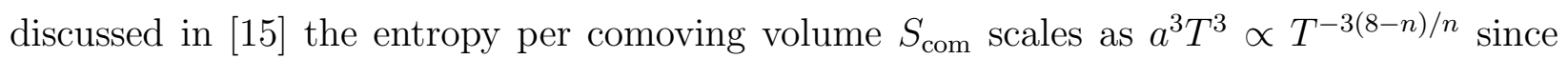
$a \propto t^{2 / n} \propto T^{-8 / n}$. Thus the dilution factor $f_{\text {dil }}$ due to entropy production between the two scales is

$$
f_{\text {dil }} \simeq\left(\frac{T_{\mathrm{dec}}}{T_{\mathrm{reh}}}\right)^{\frac{3(8-n)}{n}} .
$$

Thus there is a very significant difference between the case of the matter scaling (considered in [14]) giving $f_{\text {dil }} \simeq\left(T_{\text {dec }} / T_{\text {reh }}\right)^{5}$ and that of the kinetic mode limit with $f_{\text {dil }} \simeq T_{\text {dec }} / T_{\text {reh }}$. The origin of this difference can be easily understood: a scalar kinetic mode gets rid of most of its energy by the rapid red-shifting.

We now turn to the effect of these modifications to the pre-nucleosynthesis expansion rate on the generation of a baryon asymmetry from an $e_{R}$ asymmetry. 


\section{From $e_{R}$ to a baryon asymmetry}

Before discussing the generation of right electron asymmetry in these cosmologies, we discuss the conversion of such an asymmetry to a baryon asymmetry when $B+L$ violating processes are active. 'Conversion' is in fact a little misleading as these processes of course only act on the left-handed fermions: As will become more explicit now the physics of the creation of the baryon asymmetry is that the right electrons carry the gauge charge hypercharge, which is globally zero and exactly conserved. When there is net hypercharge in the $e_{R}$ sector, there must be also a compensating hypercharge in the rest of the particles. When this is non-zero the $B+L$ violating processes minimize the free energy with a non-zero baryon number.

We follow a standard procedure and consider the equilibrium abundance of baryon number subject to the constraints imposed by the charges conserved by the fast interactions (which are in equilibrium at that time). Because baryon number violation freezes out at the electroweak scale, when the sphaleron processes become suppressed, this is the scale at which we need to calculate baryon number. Above the electroweak scale the rate of the B-violating processes is mediated by the symmetric phase sphaleron transitions, which are unsuppressed: $\Gamma_{\mathrm{sph}} \approx 25 \alpha_{w}^{5} T \sim 10^{-6} T$ [29], so that they will have time to equilibrate above the electroweak scale in the models we are discussing ( $c f$. Eqs. (3i) and (6)). In fact we shall assume for simplicity that the expansion rate is such that the $e_{R}$ are the only standard model degrees of freedom out of equilibrium. Within the scenarios we are discussing this is not necessarily the case, as the rate could be enhanced in principle enough to take also other heavier particles out of equilibrium. For example the $\mu_{R}$ has a Yukawa coupling larger by about $10^{2}$ and therefore a decay rate faster by a factor of $\left(y_{\mu} / y_{e}\right)^{2} \sim 10^{4}$, while with a reheat temperature sufficiently close to the nucleosynthesis scale the expansion rate may be boosted in Model $A$ by almost as much as $T_{\text {ew }} / T_{\mathrm{ns}} \sim 10^{5}$, and in Model $B$ by $\left(T_{\text {ew }} / T_{\mathrm{ns}}\right)^{2} \sim 10^{10}$ times (with of course the correspondingly large entropy release factor).

With the following hierarchy of couplings

$$
\Gamma_{e_{R}} \ll H \ll \Gamma_{\mathrm{sph}}, \Gamma_{\mu_{R}}, . .
$$

the appropriate equilibrium calculation of baryon number is particularly simple. In the

standard model the only conserved charges are the gauge charges, $e_{R}$ and $\frac{1}{3} B-L_{i}$, where 
the latter is the baryon minus lepton number in each generation. We will make the slight simplification of assuming only total $B-L$ as conserved (which would be appropriate at this scale in certain models including neutrino mass matrices), which leads to minor numerical changes to the results quoted here. (We refer the reader to [8] where the full set of constraint equations can be found.)

To arrive at the set of constraint equations one expresses the charge densities in terms of particle densities $n_{\alpha}$ using $n_{\alpha}-\bar{n}_{\alpha}=\left(T^{2} / 6\right) k_{\alpha} \mu_{\alpha}$, wher@ $k_{\alpha}=1(2)$ for fermions (bosons) and $\mu_{\alpha}$ is the chemical potential for a species $\alpha$. Further $\mu_{\alpha}$ can be re-expressed in terms of the chemical potentials for charges $Q_{A}$ as follows: $\mu_{\alpha}=\sum_{A} q_{\alpha}^{A} \mu_{A}$, where $q_{\alpha}^{A}$ is the $A$-charge of the $\alpha$ species. With this we can have (cf. [8]) the following constraint equations while the baryon number is out of equilibrium:

$$
\begin{aligned}
Y & =\frac{T^{2}}{6}\left[(10+n) \mu_{Y}+8 \mu_{B-L}+\mu_{e_{R}}\right] \\
B-L & =\frac{T^{2}}{6}\left[8 \mu_{Y}+13 \mu_{B-L}-\mu_{e_{R}}\right] \\
e_{R} & =\frac{T^{2}}{6}\left[-\mu_{Y}-\mu_{B-L}+\mu_{e_{R}}\right] .
\end{aligned}
$$

Here we used the hypercharge assignments such that $Q=Y+T^{3}$, where $Q$ denotes the electric charge and $T^{3}$ the isospin. We have not written the second linearly independent gauge charge explicitly, as choosing it as $T^{3}$ it is simply proportional to its own chemical potential, and so trivially drops out of the equations when we impose $T^{3}=0$. The baryon number $B$ can itself be expressed in terms of the relevant chemical potentials as

$$
B=\frac{T^{2}}{6}\left[2 \mu_{Y}+4 \mu_{B-L}\right]
$$

The gauge charge $Y$ must be zero, and then given any value of the global conserved charges Eqs. (10) can be solved to give the baryon number (11). When $B-L$ is conserved we thus have

$$
B=\frac{2(9+2 n)}{59+12 n} e_{R}+\frac{2(11+2 n)}{59+12 n}(B-L)
$$

\footnotetext{
${ }^{7}$ We use here the massless approximation, to which there will be small corrections due to thermal masses. Note that we also assume the right electron distributions can be described by a chemical potential, which is justified given their relatively fast elastic scattering rate through weak hypercharge processes $\sim 10^{-2} T[30$.
} 
and see that $e_{R}$ is an almost equally strong source for baryon number as is $B-L$. Indeed, as $n$ changes from $n=1$ to $n=\infty$, the coefficient of $e_{R}$ changes from 0.31 to $1 / 3$, while that of $B-L$ from 0.32 to $1 / 3$. Hence Eq. (12) may be quite well approximated by

$$
B \approx \frac{1}{3}\left[e_{R}+(B-L)\right]
$$

Thus, if $B-L$ is conserved by all interactions after inflation, it is zero and remains zero, but the final baryon number, in contrast to the usual radiation dominated universe, is now non-zero and simply proportional to the original $e_{R}$ asymmetry. Indeed therefore we see

explicitly that no $B$ violation other than that of the anomalous processes of the standard model is required to produce it.

While the latter is the case which will interest us, it is interesting to note that the result that one obtains a non-zero baryon number from $e_{R}$ is very robust, and is relatively insensitive to whether the other charges are violated. Indeed it is easy to see that if $e_{R}$ is the only conserved charge $-B-L$ may for example be violated by some interactions all the way down to the electroweak scale - the net baryon number is still non-zero. Indeed, solving the reduced set of constraint equations for $Y$ and $e_{R}$ only, with $\mu_{B-L}$ set to zero, we find

$$
B=-\frac{2}{11+n} e_{R}
$$

which is slightly smaller and of the opposite sign than the result in Eq. (12).

We conclude that, irrespective of constraints on the value of $B-L$ and assumption on whether $B-L$ is conserved, a right-handed electron asymmetry is reprocessed into a baryon asymmetry of the same order.

\section{Electrogenesis}

We now consider explicitly models for electrogenesis - production of a right-handed electron asymmetry - prior to the electroweak scale. In the standard radiation dominated cosmology right electrons have been understood to be of interest because of their capacity to protect a baryon asymmetry from erasure [17, 18]. Thus their generation has been considered in the context of theories which also produce such a primordial baryon or lepton asymmetry, and 
thus typically the scale characterizing their generation is very high, around the GUT scale or in the case of leptogenesis as low as $10^{10} \mathrm{GeV}$ [31]. In the present context right electrons are in their own right adequate sources for baryogenesis by reprocessing with standard model $B+L$ violation. Given that the physics required to generate them is CP-violating only, and thus potentially can be associated in simple ways with much lower energy scales, it is certainly of interest to consider mechanisms which can produce them quite independently of $B$ or $L$ violation beyond the standard model.

In fact in the cosmologies being considered one is forced to seek such different mechanisms of $e_{R}$ generation for another very simple reason which we have not drawn attention to so far: The maximum temperature $T_{\max }$ attainable after inflation in these cosmologies is in fact much lower than in the standard radiation dominated cosmology. Given the requirements of $T_{\text {reh }}$ in (4) and (7), we can bound the temperature above by extrapolating the expansion rate to the point $H \sim 0.1 T$. For model A this corresponds to $T_{\max } \lesssim 10^{8} \mathrm{GeV}$, while for model B it gives $T_{\max } \lesssim 10^{6} \mathrm{GeV}$. Above this point thermodynamic temperature can have no meaning as the age of the Universe is shorter than the equilibration time of any process. Thus any mechanism which in the ordinary radiation dominated scenario relies on temperatures being reached higher than this is not applicable, and we must seek mechanisms which operate at a lower temperature.

Here our aim is not to be exhaustive about possible mechanisms, but rather to study an explicit model which produces an $e_{R}$ asymmetry sufficiently large to source the observed baryon asymmetry in these cosmologies. Given that in principle all the elements are present in the standard model itself, it is natural to ask - just as one does in the context of electroweak baryogenesis - whether it alone might suffice. While in the standard radiation dominated cosmology the standard model has apparently insurmountable problems on two fronts [32] the sphaleron bound and the inadequacy of standard model CP violation - here the former does not provide a significant constraint. All we require here is that the right electron number come into equilibrium after the $B+L$ violation goes out of equilibrium. This is in contrast with baryogenesis scenarios at a first order electroweak phase transition in which the sphaleron rate at the transition is required to drop below the expansion rate. So could the standard model with its CP violation produce the $e_{R}$ asymmetry? Given that its production 
can only come about through the same Yukawa coupling channel, the answer would seem to be definitively in the negative. In general however the question in these cosmologies can be framed more generally given that the expansion rate can change enormously: Is it possible to generate some CP-odd charge (not necessarily $e_{R}$ ) which is conserved on a time scale longer than that associated with the $B+L$ violating processes in the unbroken phase? We will return briefly to this question in the conclusion.

Here, just as one does in the context of baryogenesis models, we add some extra CPviolating physics in the scalar sector. We study a simple out-of-equilibrium decay of scalar particles with CP-violating decays. Interestingly we find that, again because of the modified expansion rate prior to nucleosynthesis, the mass of these scalars need not be so far above the electroweak scale for the mechanism to work. This suggests that the kind of mechanism for 'electrogenesis' we discuss may be implementated successfully in other theories with additional scalars particles at scales not far above the electroweak scale, with signatures testable at accelerators. We will return to this point in our conclusions.

\subsection{The Model}

The additional particle content we assume over the standard model (and the inflaton) is a set of Higgs-like scalar doublets $\Phi^{a}$ coupled to the standard model leptons through a Yukawa type interaction, i.e. with interaction Lagrangian

$$
\mathcal{L}_{a d d}=-h_{i j}^{a} \Phi^{a} \bar{\psi}_{i L} \psi_{j R}+h . c .,
$$

where the couplings $h_{i j}^{a}$ are CP-violating, i.e. $\mathbf{h}^{\mathbf{a} \dagger} \neq \mathbf{h}^{\mathbf{a}}$, where $\mathbf{h}^{\mathbf{a}}$ is the matrix of couplings. While in principle $\mathrm{CP}$ violation does not mandate a matrix of couplings, but only a coupling to the right electron itself with a complex phase unremovable by phase transformations on the whole Lagrangian, we will require the flavour mixing structure and the existence of at least two such scalars in order to implement the generation of a CP-violating asymmetry. The strongest constraints on the masses and the couplings of such scalars come from the fact that they are flavour changing. For leptons the strongest constraint of this type comes from the bounds on the decay $\mu \rightarrow e \gamma$ [33, 34]. For couplings $h$ of order one this requires masses $M_{\Phi} \gtrsim 100 \mathrm{TeV}$, with the branching ratio for this process going parametrically as 
$h_{\mu \tau}^{2} h_{e \tau}^{2}\left(M_{W} / M_{\Phi}\right)^{4}$ so that much smaller masses can be permitted if the couplings have a hierarchy like that in the standard model Yukawa couplings [34, 35].

\subsection{The Out-of-Equilibrium Conditions}

We consider here a simple out-of-equilibrium decay scenario for these particles, very analogous to that which occurs in standard GUT scale baryogenesis scenarios [6]. It is possible that nonperturbative decay mechanisms may be operative and work just as well, but we limit our treatment here to the simpler perturbative case. The perturbative decay rate for $\Phi$ can be well approximated by

$$
\Gamma_{\phi, \text { pert }}=\frac{|\mathbf{h}|^{2}}{8 \pi} E_{\phi}
$$

where $E_{\phi}$ is the energy of $\Phi,|\mathbf{h}|^{2}=\operatorname{Tr}\left(\mathbf{h} \mathbf{h}^{\dagger}\right)$ and we have assumed the energy of the $\Phi$ is much greater than that of the produced fermions (e.g. in the case $m_{i}=m_{j}=m$ there is a simple suppression $\left.E_{\phi} \rightarrow\left[E_{\phi}^{2}-4 m_{\psi}^{2}\right]^{1 / 2}\right)$.

Before considering the production of a CP asymmetry we first discuss the out-of-equilibrium condition. When the particles decay, with rate given by (16), the reverse process (or any other one) creating them must be suppressed. This is fulfilled if the temperature of the plasma at the time of decay is well below the mass scale of the scalars, i.e.

$$
M_{\Phi}>T, \quad \text { when } \quad \Gamma_{\phi} \sim H
$$

Equations (3) and (6) give the boost to the expansion rate with respect to the radiation dominated case as $\left(T / T_{\text {reh }}\right)^{p}$, where $p=1$ for kinetic mode domination $(n=6)$, and $p=2$ for a decaying dominant component. Making use of this and Eq. (16), we infer that the constraint $(\mathbb{1 7})$ can be re-expressed as

$$
M_{\Phi}>T_{\mathrm{dec}}>\left(70 g_{*}\right)^{-1 / 2(1+p)}\left[|\mathbf{h}|^{2} M_{P} T_{\mathrm{reh}}^{p}\right]^{\frac{1}{1+p}} \quad(0 \leq p \leq 2),
$$

where $T_{\text {dec }}$ is the temperature at which $\Phi$ decays, and we have used $H_{\text {rad }}=\left(\pi^{2} g_{*} / 90\right)^{\frac{1}{2}} T^{2} / M_{P}$ (where $M_{P} \approx 2.4 \times 10^{18} \mathrm{GeV}$ ). For the case of radiation domination $(p=0)$ this gives $M_{\Phi}>T_{\text {dec }}>10^{16}|\mathbf{h}|^{2} \mathrm{GeV}$, where we took $g_{*} \sim 10^{3}$. Given that in these scenarios the asymmetry is generated by, at the very least, the interference between a tree-level and oneloop diagram, it is always suppressed by some small numbers times at least a square of the 
couplings $\mathbf{h}$, and often by higher powers of the couplings. Hence to produce a significant asymmetry one cannot have the coupling too small, and conversely one needs the scalar field to have a mass not so far below the GUT scale.

For the cosmologies we are primarily considering these bounds change very considerably. In Model $A$ in which the Universe is dominated by a kinetic mode ( $p=1$, or equivalently $n=6)$ the constraint (18) relaxes to

$$
M_{\Phi}>T_{\mathrm{dec}}>3 \times 10^{6} \mathrm{GeV}|\mathbf{h}|\left(\frac{T_{\mathrm{reh}}}{T_{\mathrm{ns}}}\right)^{\frac{1}{2}}>5|\mathbf{h}| \times 10^{6} \mathrm{GeV} .
$$

where we took $T_{\mathrm{ns}}=1 \mathrm{MeV}$ and $g_{*} \sim 10^{3}$. This should be compared with the energy scale $H_{I}$ which characterizes this model at the beginning of the post-inflationary epoch. For a reheat temperature $T_{\text {ns }}$ and a pure $n=6$ scaling after inflation one finds [8]

$$
H_{I} \sim 10^{7} \mathrm{GeV}\left(\frac{\mathrm{T}_{\mathrm{reh}}}{\mathrm{T}_{\mathrm{ns}}}\right)^{\frac{1}{2}}
$$

Thus the $M_{\Phi}$ can be sufficiently light that they are produced by gravitational coupling in this mechanism along with all the other lighter $(m<H)$ degrees of freedom. A little later, at a temperature $T \sim 0.1 H_{I}$ the strongly interacting degrees of freedom begin to equilibrate (and define a real thermodynamic temperature), while the $\Phi$ can decay without ever coming into equilibrium. For smaller values of the coupling $\left(h \lesssim 10^{-2}\right)$ there may be some time for weak force mediated annihilation processes (with rate $\sim \alpha_{w}^{2} T$ ) to act, and in this case the initial $e_{R}$ number density at the time of decay will be reduced somewhat relative to their initial value.

In the case of Model B, when the dominant component decays, we have $p=2$ so that Eq. (18) gives an even milder bound on the mass of $\Phi$ :

$$
M_{\Phi}>T_{\mathrm{dec}}>2|\mathbf{h}|^{\frac{2}{3}}\left(\frac{T_{\mathrm{reh}}}{T_{\mathrm{ns}}}\right)^{\frac{2}{3}} \mathrm{TeV}>3|\mathbf{h}|^{\frac{2}{3}} \mathrm{TeV} .
$$

In this case therefore the out-of-equilibrium condition may in some cases (for sufficiently low $\left.T_{\text {reh }}\right)$ provide an even weaker constraint on their masses than accelerator constraints from the flavour changing processes they can mediate. More generally, it is certainly interesting to note that the mass scale is sufficiently low that models may be viable in which the scalars 
are the supersymnmetric scalar partners of the standard model particles. We will return to this point in our conclusions. Therefore in models of type $B$ we can envisage the following scenario. The universe attains a temperature $T \gg M_{\Phi}$ and the $M_{\Phi}$ are created by the fastest

processes in similar quantities to the other degrees of freedom; as the temperature falls they drop out of equilbrium and, when the temperature $T_{\mathrm{dec}}$ is reached, they decay. As in Model $A$ one would need to consider carefully the different cases (depending on $|\mathbf{h}|$ ) in which the weak interactions can or cannot play a role in reducing the particle anti-particle asymmetry in $\Phi$ before this decay occurs. One feature of (21) should immediately be noted, however, and we will return to it below: The entropy release of these models which is of relevance in the present case is that which occurs between the time of production of the $e_{R}$ asymmetry, $T_{\mathrm{dec}}$, and $T_{\text {reh }}$. From (21) it follows that

$$
\frac{T_{\mathrm{dec}}}{T_{\mathrm{reh}}}>2|\mathbf{h}|^{\frac{2}{3}}\left(\frac{\mathrm{TeV}}{T_{\mathrm{ns}}^{2 / 3} T_{\mathrm{reh}}^{1 / 3}}\right) \gtrsim 10^{5}|\mathbf{h}|^{\frac{2}{3}},
$$

where the latter equality follows from (7). When it comes to producing a final baryon asymmetry this constraint will make it very difficult for models with any scaling much slower than the kinetic mode $\left(\rho_{\phi} \propto a^{-6}\right)$ to produce a reasonable final baryon to entropy ratio. We will return to this below.

\subsection{Generation of the Asymmetry}

We now turn to the production of the asymmetry through the out-of-equilibrium decay of these scalar fields. As in any CP-violating out-of-equilibrium decay scenario one must go beyond the tree-level decay and consider the interference between tree-level and higher order diagrams to produce any net CP-violating effect. Further, since CPT theorem implies the equality between the total decay rate for particles and anti-particles, we need at least two channels containing different $e_{R}$ number in order to be able to produce the asymmetry. It is to this end that we have taken the $\Phi^{a}$ scalars to couple to more than one generation. Further, to produce a CP-violating effect at one loop level we need at least two scalars, just as one requires two heavy bosons in simple GUT scenarios ( $c f$. Ref. [6]). Here we do not try to be exhaustive in our consideration of the diagrams which give dominant contributions in different parts of parameter space. In Figure 2 the two diagrams we consider here are shown, 

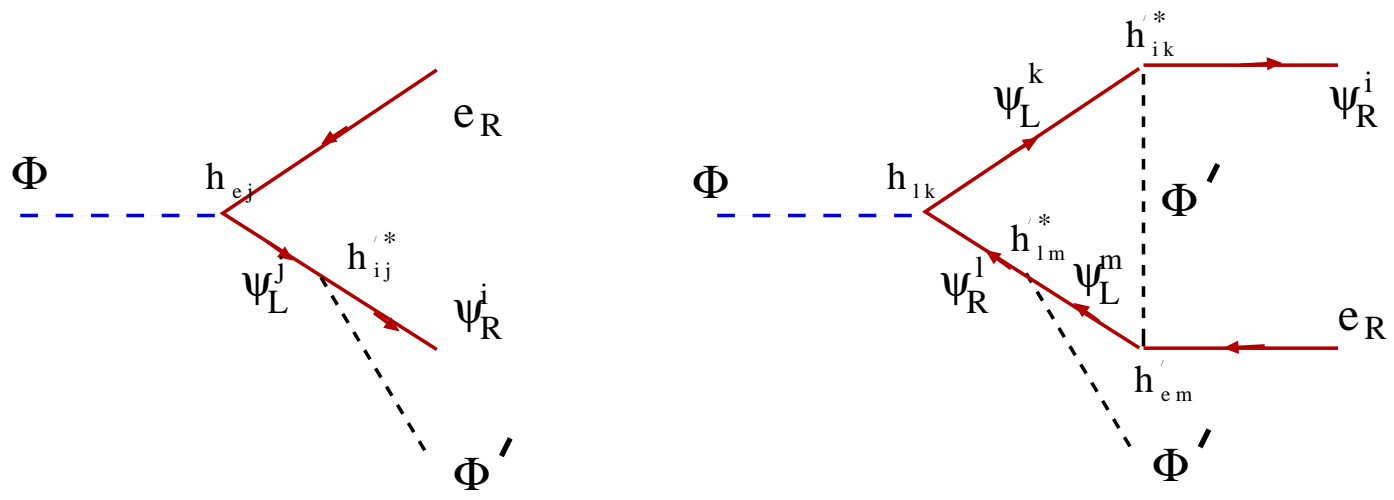

Figure 2: Tree and one loop diagrams for the three body decay $\Phi \rightarrow \bar{e}_{R} \Psi_{R}^{i} \Phi^{\prime}$, with the appropriate couplings at the vertices. We assume that $\Phi$ is heavier than $\Phi^{\prime}$. When the second outgoing lepton is a $\mu$ or $\tau$ lepton the process produces net $e_{R}$ number.

for the decay channel $\Phi \rightarrow \bar{e}_{R} \Psi_{R}^{i} \Phi^{\prime}$ where the $\Phi^{\prime}$ is assumed to be the lighter of the (at least) two scalars. Provided the second fermion $\Psi_{R}^{i}$ is of one of the two heavier lepton flavours, the process violates right electron number. The rate of the corresponding anti-particle decay does not cancel if the CP-violating interference terms between the two diagrams have a pure complex part. Summing over the internal fermions we have (see [36] for somewhat similar cases) that the net CP-violating effect creating net $e_{R}$ number compared to the tree-level decay is

$$
\frac{\Gamma_{\Phi \rightarrow \bar{e}_{R} \Psi_{R}^{i} \Phi^{\prime}}-\Gamma_{\bar{\Phi} \rightarrow e_{R} \bar{\Psi}_{R}^{i} \bar{\Phi}^{\prime}}}{\Gamma_{\Phi}^{t o t}+\bar{\Gamma}_{\bar{\Phi}}^{t o t}}=\epsilon_{p} \frac{\operatorname{Im}\left[\left(\mathbf{h h}^{\prime \dagger}\right)_{e i}\left(\mathbf{h}^{\prime} \mathbf{h}^{\dagger} \mathbf{h}^{\prime} \mathbf{h}^{\prime \dagger}\right)_{i e}\right]}{\operatorname{Tr}\left[\mathbf{h} \mathbf{h}^{\dagger}\right]}
$$

where $\epsilon_{p} \sim 10^{-2}$ is the phase space factor (and $i$ is summed over the non-electron indices). There is also another pair of diagrams which differ only in that the $\Phi^{\prime}$ emission on the external $e_{R}$ leg, which gives (23) with the indices interchanged. From the result we see clearly that to obtain an effect at this order we indeed need two scalars since, when $\mathbf{h}=\mathbf{h}^{\prime}$, the result in Eq. (23) vanishes. We note that a diagram in which the $\Phi^{\prime}$ on the external leg is the standard model Higgs could dominate if the $\mathbf{h}^{\prime}$ couplings are all smaller than the Yukawa coupling of the $\tau$ lepton $\left(y_{\tau} \approx 10^{-2}\right)$.

We thus write the resultant $e_{R}$ asymmetry as

$$
\frac{e_{R}}{s}\left(T_{\mathrm{dec}}\right) \sim \frac{10^{-2}}{g_{*}}|\mathbf{h}|^{4} \delta_{C P}
$$


where $\delta_{C P}$ is proportional to the imaginary part in (23). If all the couplings $h$ are of the same order this can be of order one, while if there is a hierarchy similar to that in the standard model, it will be correspondingly smaller.

\subsection{The Baryon Asymmetry}

To arrive at the final baryon asymmetry the cases of Model $A$ and Model B are quite different. In the former case there is no entropy dilution and the final baryon to entropy ratio is quite simply given by (13), i.e. one third of the right electron to entropy ratio (24). We thus have

$$
\frac{n_{B}}{s} \sim \frac{10^{-2}}{g_{*}}|\mathbf{h}|^{4} \delta_{C P}, \quad(\text { Model } A),
$$

which for roughly equal on- and off-diagonal couplings in $\mathbf{h}$ and $\mathbf{h}^{\prime}$ gives a baryon asymmetry of the required size $n_{B} / s \sim 10^{-10}$ for couplings of the order $\sim 10^{-1}-10^{-2}$. Note that this corresponds (from (19)) to scalar masses as low as about $M_{\phi} \sim 100 \mathrm{TeV}$.

In Model $B$ there is the important entropy dilution factor, so that for the final baryon asymmetry we find

$$
\frac{n_{B}}{s} \sim \frac{10^{-2}}{g_{*}}|\mathbf{h}|^{4} \delta_{C P}\left(\frac{T_{\mathrm{reh}}}{T_{\mathrm{dec}}}\right)^{\frac{3(8-n)}{n}}, \quad(\text { Model } B) .
$$

If we take the constraint given in (22) for couplings and $\delta_{C P}$ of order one, we can have a baryon asymmetry compatible with that required for nucleosynthesis only for a case $n \approx 6$, i.e. when the inflaton rolls in a kinetic energy dominated mode while it decays. The outof-equilibrium decay condition for the $\Phi$ field (21) is in this case satisfied for $M_{\phi}$ as small as a few $\mathrm{TeV}$. However the constraints on the flavour changing neutral currents need to be carefully considered, but can still be satisfied (e.g. if one of the couplings $h_{e \mu}$ or $h_{e \tau}$ is much smaller than the others). This provides in principle an interesting probe at accelerators of these models in a parameter range which is of interest.

For the standard matter scaling $(n=3)$ [14, or indeed radiation scaling $(n=4)$ during

reheating, the entropy dilution factor in (26) is much too large to allow the generation of the required baryon asymmetry, and the mechanism we have discussed is not a viable one for baryogenesis in these cases. In a different model it may be possible to relax the condition (12) and reduce the dilution factor. This would be appropriate for example if the $e_{R}$ continues 
to be created all the way down to the electroweak scale, for example in a scenario in which the $\Phi$ particles are themselves directly produced out of equilibrium by the inflaton decay all the way to that scale. Tuning the value of $T_{\text {reh }}$ to be just enough to keep the $e_{R}$ out of equilibrium until that time, i.e. to satisfy (可), the case $n=3$ gives a dilution by a factor $\sim 10^{6}$, and the case $n=3$ by $\sim 10^{4}$. A fairly copious initial $e_{R}$ asymmetry must therefore be produced very close to the electroweak scale in order to give the required baryon asymmetry.

The model we have presented here is completely perturbative. It is likely that there are models of non-perturbative decay of a condensate of the $\Phi$ field in which the constraints inferred on the Yukawa coupling $\mathbf{h}$ may be relaxed. One simple possibility would be a variant of the well-known Affleck-Dine mechanism [37], with a scalar field $\Phi$ charged under right-handed electron number, which oscillates and decays. This may occur for example in

supersymmetric extensions of the Standard Model when an $A \Phi^{3}+$ h.c. term is present in the potential for a weakly coupled scalar field. When the field decays it creates a net right-handed electron number which is not suppressed by any coupling constant. The suppression may also potentially be evaded without the scalar field being required to carry a right electron charge. This would occur if there were a resonant decay into the electrons, which may in fact occur through precisely the same Yukawa coupling discussed here. Because they do not have the Yukawa coupling suppression, these mechanisms for producing $e_{R}$ would leave more space for entropy release in models like our model B. However, as we have pointed out, this will only work in the special case that the $e_{R}$ is created very close to the electroweak scale, and the temperature at which radiation domination begins $T_{\text {reh }}$ lies just far enough below the electroweak scale to keep the $e_{R}$ out of equilibrium at the electroweak scale.

\section{Anomaly and Magnetic Fields}

So far we have neglected the effects related to the abelian anomaly discussed in the introduction. As described in [16 the effect of this term on a finite chemical potential $\mu_{R}$ for right electrons is to cause an instability in modes with $k<\mu$. Naively this instability can begin to grow when the corresponding mode enters the horizon (i.e. $\mu \sim H$ ), but when the slowing effect of conductivity $\sigma$ in the plasma is taken into account the criterion becomes 
$\mu^{2} / \sigma \sim H$. In the standard case, in which the mode must be able to start to evolve before the perturbative $e_{R}$ decay comes back into play, this corresponds to the effect being important if $\mu / T \gtrsim 10^{-6}$. Here since the expansion rate is such that the $e_{R}$ come back into equilibrium below the electroweak scale, the lower bound (which depends now simply on the expansion rate at the electroweak scale) will in fact be the same or larger. Moreover, while in the standard case the effect of the perturbative channel is important, and leads to the requirement that a significantly larger asymmetry than the critical one be present initially in order for the source hypermagnetic field to survive to the electroweak scale, here the instability will simply develop all the way to that scale and there will be no damping of the fields due to the appearance of the perturbative channel.

The conclusions we can draw are as follows:

- Model A: If the initial $e_{R}$ asymmetry is less than the critical value for hypermagnetic field generation, all our analysis given above holds. A baryon asymmetry is produced of the same order, and thus for compatibility with the observed asymmetry one must have the corresponding initial value $\mu_{R} \sim 10^{-10} \mathrm{~T}$. For initial $\mu_{R}$ larger than the critical value, hypermagnetic field will be generated. The baryon asymmetry generated will depend on the attained value of the chemical potential $\mu_{R}$ (which reduces as the Chern-Simons number grows in the condensate). However, the latter will always be bounded below by the same critical value, and thus the baryon asymmetry also (see also 19 for a discussion of the full evolution of the dynamical equations). Thus we conclude that magnetic field generation from $\mu_{R}$ cannot be attained in this model with an altered expansion rate, since it will always be associated with a baryon asymmetry which is too large. On the other hand, the baryogenesis mechanism from right electrons works perfectly well, and is unaffected by the anomalous effects as the chemical potential is so small.

- Model B: The effects of the interplay of the baryon generation and the instability causing the growth of magnetic field are much more difficult to evaluate in this case, and are in general model dependent. The fact that the entropy dilution effect mandates a larger initial electron asymmetry, which would then be subject to the instability, suggests that it may be possible to find a model in which both magnetic field and the observed baryon asymmetry could be produced from the right electrons. As was discussed above, the production of the baryon 
asymmetry would require that a large $\mu_{R}$ chemical potential be produced very close to the electroweak scale. The corollary is that, if it is produced close to the electroweak scale, there will be little time for the instability to evolve and create significant seed fields. One way of getting around this would be in a model with a continuous sourcing of the $e_{R}$ asymmetry, so that the contribution at earlier time may grow into a magnetic field. The driving chemical potential is, however, itself being constantly diluted by entropy production, and the energy in the resultant field also relative to the background energy density. To see whether seed fields of significant magnitude can survive to the electroweak scale would require detailed study in a model of $e_{R}$ generation quite different to that we have discussed.

\section{Conclusions}

We have considered here one aspect of cosmologies in which a scalar field dominates the expansion rate prior to nucleosynthesis. Right-handed electrons may remain out of equilibrium until the electroweak scale, so that if they are generated the $B+L$ processes of the standard model will lead to a non-zero equilibrium density of baryons of the order of that in the $e_{R}$. We have discussed two kinds of post-inflationary cosmologies in which such a period of scalar field domination can occur: in the first the inflaton rolls away after inflation into a kinetic mode in a steep potential, and the Universe is 'reheated' by the gravitational particle production at the end of the inflationary epoch, while in the second the inflaton rolls into a mode which can have a range of scalings and reheats the Universe itself by decaying sufficiently slowly to give a very low reheat temperature. We studied a specific model for the generation of the right handed electron asymmetry in which there are a set

of scalars with CP-violating (and flavour changing) couplings to the leptons. We showed that in both scenarios such scalars can decay out of equilibrium at quite low temperatures and produce the desired asymmetry. While our models strongly favoured the case of kinetic mode domination, which have little or no entropy release, we note that in certain very special circumstances which may be satisfied in other models the generation of the observed baryon asymmetry may still be possible in the standard reheating scenario (with matter scaling during the reheating epoch). Finally we considered briefly the effect of the abelian anomaly 
which destabilizes such charges, and concluded that in the models with kinetic mode domination this effect is unimportant for the baryon number generation, while in the case with large entropy dilution it may be important and might allow the generation of magnetic field as in the case of standard radiation domination.

Finally we return to the question of how this kind of mechanism might be implemented in other particle physics models, in particular in more popular (e.g. supersymmetric) extensions of the standard model. In general one need not consider necessarily the generation of right-handed electron number, but the generation of any CP odd charge which is effectively conserved after its creation on a timescale which is longer than the expansion rate of the Universe at the electroweak scale (when the $B+L$ violating processes freeze-out). Given that the expansion rate at the electroweak scale can be enhanced in these models by many orders of magnitude - up to a rate $\sim 10^{-11} T_{\text {ew }}$ in models of type $\mathrm{A}$, and $\sim 10^{-6} T_{\text {ew }}$ in models of type B - scenarios can be considered in which many of the lighter degrees of freedom will drop out of equilibrium (for example the lighter right-handed quarks). While in the standard model itself there would seem to be the obstacle of prohibitively small CP violation, in extensions there is generically new CP-violating structure in the added sectors (e.g. in the chargino and squark mass matrices of the minimal supersymmetric standard model). The

problem of baryogenesis then becomes the problem of the generation prior to the electroweak scale of CP-odd approximately conserved charge using this structure. Given our observation that for very modest masses (as low as a $\mathrm{TeV}$ for a particle with a coupling of order one) the decay of these heavier particles occurs out of equilibrium in these cosmologies, there is clearly the interesting possibility of sourcing CP-odd charges in this way, thus creating a baryon asymmetry. We will treat these issues in detail in forthcoming work [38].

\section{Acknowledgements}

We would like to thank Kimmo Kainulainen and Misha Shaposhnikov for useful discussions. MJ thanks Sacha Davidson and Steve Abel for discussion while this work was being completed. 


\section{References}

[1] Supernova Cosmology Project (S. Perlmutter et al., Nature 391, 51 (1998), astroph/9712212; S. Perlmutter et al., Astrophys. J. 516 (in press), astro-ph/9812133; A.G. Riess et. al., Astron. J. 116, 1009 (1998).

[2] See for example L. Wang, R.R. Caldwell, J.P. Ostriker and P.J. Steinhardt, Astrophys. J. 530, 17 (2000), astro-ph/9901388; I. Waga and J.A. Frieman, astro-ph/0001354.

[3] P. Ferreira and M. Joyce, Phys. Rev. Lett. 79, 4740 (1997), astro-ph/9707286; P. Ferreira and M. Joyce, Phys. Rev. D58, 023503 (1998), astro-ph/9711102.

[4] I. Zlatev, L. Wang and P.J. Steinhardt, Phys. Rev. Lett. 82, 896 (1999), astroph/9807002; P.J. Steinhardt, L. Wang and I. Zlatev, Phys. Rev. D59, 123504 (1999), astro-ph/9812313.

[5] See for example P. Kernan and S. Sarkar, Phys. Rev. D54, 3681 (1996); B.D. Fields, K. Kainulainen, K.A. Olive and D. Thomas, New. Astron 1, 77 (1996).

[6] E. Kolb and M.S. Turner, The Early Universe (Frontiers in Physics, vol. 69), AddisonWesley Publishing Company (1990).

[7] B. Spokoiny, Phys. Lett. B315, 40 (1993).

[8] M. Joyce and T. Prokopec, Phys. Rev. D57, 6022 (1998), hep-ph/9709320.

[9] P.J.E. Peebles and A. Vilenkin, Phys. Rev. D59, 063505 (1999), astro-ph/9810509.

[10] M. Joyce, Phys. Rev. D55, 1875 (1997), hep-ph/9606223.

[11] J.D. Barrow, Nucl. Phys. B208, 501 (1982).

[12] M. Kamionkowski and M.S. Turner, Phys. Rev. D42, 3310 (1990); K. Griest, M. Kamionkowski and M.S. Turner, ibid. 41, 3565 (1990).

[13] L.H. Ford, Phys. Rev. D35, 2955 (1987). 
[14] S. Davidson, M. Losada, and A. Riotto, hep-ph/0001301.

[15] T. Prokopec, hep-ph/0002181.

[16] M. Joyce and M.E. Shaposhnikov, Phys. Rev. Lett. 79, 1193 (1997), astro-ph/9703005.

[17] B. Campbell, S. Davidson, J. Ellis and K. Olive, Phys. Lett. 297B, 118 (1992).

[18] J.M. Cline, K. Kainulainen and K.A. Olive, Phys. Rev. Lett. 71, 2372 (1993), hepph/9304321; J.M. Cline, K. Kainulainen and K.A. Olive, Phys. Rev. D49, 6394 (1994), hep-ph/9401208.

[19] M. Giovannini and M.E. Shaposhnikov, Phys. Rev. Lett. 80, 22 (1998), hep-ph/9708303 and Phys. Rev. D57, 2186 (1998), hep-ph/9710234.

[20] J.J. Halliwell, Phys. Lett. B185, 341 (1987); J.D. Barrow, Phys. Lett. B187, 341 (1987).

[21] B. Ratra and P.J.E. Peebles, Phys. Rev. D37, 3406 (1988).

[22] R.R. Caldwell, R. Dave and P.J. Steinhardt, Phys. Rev. Lett. 80, 1582 (1998), astroph/9708069.

[23] M.S. Turner, Phys. Rev. D28, 1243 (1983).

[24] M. Dine, hep-th/0002047.

[25] M. Giovannini, Phys. Rev. D60, 123511 (1999), astro-ph/9903004.

[26] Francesca Rosati, Talk given at COSMO 99: 3rd International Conference on Particle Physics and the Early Universe, Trieste, Italy, 27 Sep - 3 Oct 1999, hep-ph/0002090; M. Peloso and F. Rosati, JHEP 9912, 026 (1999), hep-ph/9908271.

[27] E. W. Kolb, A. Riotto and I. I. Tkachev, Phys. Lett. B423, 348 (1998), hep-ph/9801306.

[28] G. Felder, L. Kofman and A. Linde, Phys. Rev. D60, 103505 (1999), hep-ph/9903350. 
[29] D. Bodeker, G. D. Moore, K. Rummukainen, Phys. Rev. D61, 056003 (2000), hepph/9907545; G. D. Moore, hep-ph/0001216.

[30] M. Joyce, T. Prokopec and N. Turok, Phys. Rev. D53, 2930 (1996).

[31] See for example W. Buchmuller and S. Fredenhagen, Presented at International School of Astrophysics, Daniel Chalonge: 7th Course: Current Topics in Astrofundamental Physics (A NATO Advanced Study Institute Euroconference), Erice, Italy, 5-16 Dec 1999; hep-ph/0001098; W. Buchmüller and M. Plümacher, contribution to the Festschrift for L.B. Okun, submitted to Phys. Rep., hep-ph/9904310.

[32] See for example V. Rubakov and M.E. Shaposhnikov, Phys. Usp. 39, 461 (1996).

[33] M. Sher and Y. Yuan, Phys. Rev. D44, 1461 (1991).

[34] M. Sher, in the Proceedings of 29th International Conference on High-Energy Physics (ICHEP 98), Vancouver, Canada, 23-29 Jul 1998, hep-ph/9809590.

[35] A. Antaramian, L.J. Hall and A. Rašin, Phys. Rev. Lett. 69, 1871 (1992).

[36] T. Yanagida and M. Yoshimura Phys. Rev. D23, 2048 (1981); M. Fukugita and T. Yanagida, Phys. Lett. B174, 45 (1986).

[37] I. Affleck and M. Dine, Nucl. Phys. B249, 361 (1985); M. Dine, L. Randall and S. Thomas, Nucl. Phys. B458, 291 (1996), hep-ph/9507453. B.A. Campbell, M.K. Gaillard, H. Murayama and K.A. Olive, Nucl. Phys. B538, 351 (1999), hep-ph/9805300.

[38] M. Joyce and T. Prokopec, in preparation. 